\title{
IMPORTÂNCIA DA DIETA NA EPIDEMIOLOGIA DO CÁNCER DE COLON E RETO
}

\author{
Eliza da Conceição da Fonseca Lopes* \\ Sandra Casa Nova Derivi** \\ Maria Heidi Marques Mendez**
}

LOPES, E. da C. da F. et al. Importância da dieta na epidemiologia do câncer de colon e reto. Rev. Saúde públ., S. Paulo, 18:405-10, 1984.

\begin{abstract}
RESUMO: Foi feita atualização de estudos realizados com o objetivo de verificar a relação entre o fator ambiental, destacando-se a alimentação caracterizada por uma dieta pobre em fibra e rica em gordura e a distribuiçáo epidemiológica do câncer de colon e reto. Sáo enfatizadas as diferenças apresentadas pelas dietas dos países industrializados e dos países em desenvolvimento $\mathrm{e}$ a influência da religito e do fluxo migratório no hábito alimentar, associadas com as taxas de incidência da doença.
\end{abstract}

UNITERMOS: Epidemiologia. Câncer de colon. Câncer de reto. Hábitos alimentares. Dieta.

Os estudos que vêm sendo realizados por vários autores apontam o fator ambiental, onde destaca-se a dieta habitual das populaçб̃es, desempenhando importante fator no aparecimento do câncer de colon e reto 4,8 , $9,11,14,19,32$.

Alguns autores $4,25,36,37,38$ explicam a patogênese do câncer de colon e reto em relação à dieta, devido a acentuada influência exercida na atividade da microflora intestinal onde a presença de dietas ricas em gordura e pobres em "fibra da dieta" favorecem a proliferação bacteriana e a conseqüente degradação dos ácidos biliares produzindo agentes carcinogênicos potenciais. A presença de dietas pobres em fibra provoca retardamento da função intestinal ocasionando prolongamento de tempo de contato com a mucosa intestinal de agentes carcinogênicos presentes no conteúdo fecal ${ }^{3}, 7,36$.

A predominância de tumores do intestino grosso ocorre nas partes onde as fezes ten. dem a estagnar, correspondendo ao início do colon ascendente, colon distal e reto. Este fato sugere a relação entre a indução do tu. mor e o contato prolongado entre as fezes e a mucosa intestinal ${ }^{13}$.

Pesquisas realizadas enfatizam a raridade de enfermidades do trato intestinal, destacadamente hemorróides, colite ulcerativa, apendicite, diverticulite e câncer de colon e reto, em comunidades rurais Africanas ${ }^{2}, 24,31$. Burkitt ${ }^{5,6}$ relacionou a presença dessas doenças com o tipo de alimentação das popula. çóes e observou que as tribos africanas que consomem dietas ricas em fibra $(60 \cdot 170 \mathrm{~g}$ de fibra da dieta) apresentam baixa incidên. cia da doença. Nos países em desenvolvimen. to, o consumo de alimentos glicídicos inte. grais tendem a reduzir à medida em que o processo de urbanização vai alcançando estágios mais avançados, ocasionando uma diminuição nos teores de fibra na dieta.

Burkitt e col. ${ }^{7}$ e Southgate e col. ${ }^{29}$ apresentam análise comparativa do consumo alimentar verificado na inglaterra, Estados Uni-

* Do Departamento de Nutrição da Universidade Federal Fluminense - Rua Jansen de Melo, 174 24220 - Niterói, RJ.

* Do Departamento de Farmácia da Universidade Federal Fluminense - Rua Dr. Mario Viana, 523 Santa Rosa - 24220 - Niterói, RJ. 
LOPES, E. da C. da F. et al. Importância da dieta na epidemiologia do câncer de colon e reto. Rev. Saúde públ., S. Paulo, 18:405-10, 1984.

dos e Alemanha, observando uma mudança de alimentação nestes últimos 100 anos, com um acentuado declínio no consumo de fibra. Foi verificada reduçâo no consumo de farinha de trigo e dos grãos de um modo geral, de $43 \%$ para $13 \%$.

Heller e Hackler ${ }^{16}$ e Robertson ${ }^{26}$ fazem referencia ao fato de que a introdução do processo de moagem entre 1877 e 1880 , o crescimento do processo industrial dos alimentos e as mudanças oriundas da tecnolo. gia, foram fatores que contribuíram para uma queda no consumo da "fibra da dieta". Heller e Hackler ${ }^{16}$ mostram que o consumo de "fibra da dieta" nos Estados Unidos, proveniente de cereais, feijoes, vegetais e frutos, manteve-se elevado no período de guerra $e$ pós-guerra, decaindo progressivamente nos anos seguintes, quando, no período de 1957 a 1959 , atingiu uma queda de $28,0 \%$ declinando até um consumo diário de $4,8 \mathrm{~g}$ de fibra bruta, per capita, que permaneceu até 1970.

Trabalho realizado nos Estados Unidos ${ }^{15}$, comparando o teor de fibra presente na dieta de vegetarianos puros e ovo-lacto vegeta. rianos, mostrou um consumo de altos teores de fibra nestes grupos. Este dado relacionado com a ausência de distúrbios gastrintestinais demonstrou existir relação positiva en. tre o alto consumo de fibra e a ausência de doenças intestinais nos dois grupos de vegetarianos.

Walker e col. ${ }^{33}$ (1982) mostraram que o consumo de fibra da dieta na alimentação, em períodos de proximidade à II Guerra Mundial, eram procedentes dos seguintes alimentos: pão de alta extração, batatas e vegetais, sendo que este consumo contribui com o dobro do consumo de "fibra da dieta" verificado no período de paz, atingindo $30-40$ $\mathrm{g}$ de fibra por dia, refletindo em melhoria geral da saúde da população.

Segundo Howell ${ }^{18}$, a American Cancer Society, no período de 1959 a 1960 , realizou pesquisa sobre os hábitos alimentares, em indivíduos de 40 a 79 anos, nos Estados Unidos. Foi verificada a diferença entre os alimentos consumidos pela população do norte e do sul do país.
A do norte apresentou menor consumo de arroz em relação ao sul e, até 1969 , essa situação permaneceu inalterada, sendo constatada em maior extensão, a predominância do câncer do colon e reto no norte em relação ao sul.

Os países do norte da Europa apresentam, em geral, baixa incidência de câncer de colon e reto, registrando-se na área rural de Kuopio (Finlândia) as menores taxas. Neste estudo ${ }^{10}$ observou-se na Dinamarca (Copenhagen) baixo consumo de feijão, cereais integrais, batatas e leite, e consumo elevado de carnes; en. quanto que a região rural de Kuopio apresentou elevado consumo de cereais integrais, frutas, vegetais e leite. $O$ consumo de "fibra da dieta" nesta região, é elevado em comparação aos países de risco: Dinamarca $(17,2 \mathrm{~g} /$ dia); Inglaterra (19,9 g/dia) e Alemanha (24 g/dia), e aproximando-se dos resultados da Suiça e Inglaterra no període de 1942-1950, cujo consumo diário varia de $30-40 \mathrm{~g}$, tendo sido associado a este fato uma acentuada melhoria na saúde da população, principalmen. te aquela relacionada ao trato gastruntesti$\mathrm{nal}^{28,33}$.

A tabela mostra a incidência de câncer de colon e reto em vários países e populações.

Observamos pelos dados apresentados na Tabela que a incidência de casos de câncer de colon e reto apresenta-se bem mais eleva. da nos países industrializados que nos países em desenvolvimento. Os dados coletados por Burkitt ${ }^{4}$ demonstraram que a freqüência dos casos de câncer de colon e reto, nos países do Sul da África, variam em torno de 0,2 a $4,4 \%$ do total de carcinomas. Burkitt ${ }^{4}$ cita também que somente 2 casos de câncer de colon e reto foram observados em mais de 40.000 espécimes cirúrgicas realizadas na Uganda e que na Rodésia, em mais de 1.300 autópsias realizadas, não foi encontrado nenhum caso de câncer do intestino grosso.

No Brasil, poucos são os trabalhos desenvolvidos posicionando a incidência dessas doenças em âmbito nacional.

Souza $^{29}$ (1982) apresenta estudo sobre as 10 principais causas de mortalidade por neoplasias malígnas no ano de 1978, no Estado de São Paulo. Esse estudo apontou o câncer 
LOPES, E. da C. da F, et al. Importância da dieta na epidemiologia do câncer de colon e reto. Rev. Saúde públ., S. Paulo, 18:405-10, 1984.

TABELA

Incidência do câncer de colon e reto por 100.000 hab. (Taxas ajustadas pela população mundial)

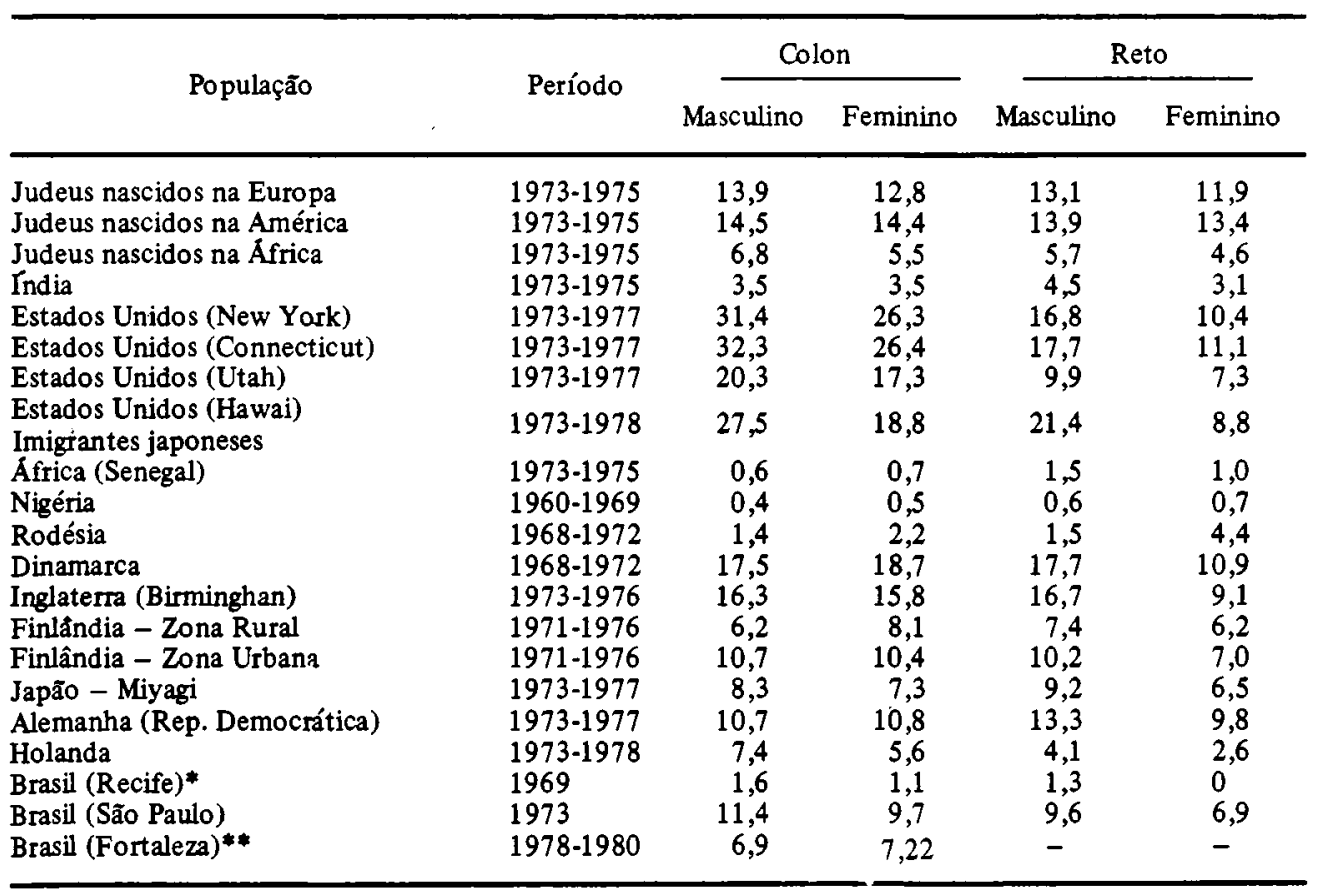

Fontes: * Waterhouse, J. C. et al. ${ }^{34,35}(1976,1982)$

** Silva, M.G.C. $\mathrm{da}^{27}$ (1982)

do intestino grosso ocupando lugar de destaque: 06 ? lugar para o sexo masculino e o 3 ? lugar para o sexo feminino.

Estudo realizado por Barcelos e Peccin ${ }^{1}$ (1983), no Rio Grande do Sul, apontou o câncer de colon ocupando o 10 \% lugar para o sexo masculino e $6 \%$ lugar para o sexo feminino, como causa de mortalidade por neo. plasias malígnas.

Em 1982, a Campanha Nacional de Combate ao Câncer ${ }^{23}$ publicou estudo realizado sobre as 10 principais localizações de câncer no Brasil, no período de 1976-1980. Observou-se que a distribuição percentual dos diagnósticos histopatológicos do câncer do intestino grosso (no sexo mas. culino) está entre as 10 principais localizações de câncer primário no Brasil, encontran. do-se em 4 9 lugar no Estado de São Paulo, seguido dos Estados do Rio de Janeiro e Mato Grsso do Sul, em 6? lugar; e nos Estados do Amazonas, Minas Gerais, Mato Grosso, Distrito Federal, Ceará, Rio Grande do Norte, Bahia, Pará e Goiás, em 7 ? lugar; sendo que os demais Estados ocupam posiçбes mais distantes entre o 8 ? e 10 ? luga. res; refletindo em menores percentuais na região norte e nordeste.

Os dados referentes a situação do câncer de intestino grosso comparada a outras loca. lizaçбes anatômicas, para o sexo feminino, encontram-se no 40 lugar nos Estados da Bahia, Mato Grosso do Sul, São Paulo, Rio de Janeiro e Rio Grande do Sul; seguido dos Estados do Amazonas, Santa Catarina, Distrito Federal, Minas Gerais, Paraná, Parafba 
LOPES, E. da C. da F. et al. Importância da dieta na epidemiologia do câncer de colon e reto. Rev. Saúde públ., S. Paulo, 18:405-10, 1984.

e Pernambuco, em 59 lugar; nos Estados do Ceará, Espirito Santo, Goiás, Sergipe, Pará e Piauí, em 6? lugar e, em 7 ? lugar, o Estado do Rio Grande do Norte, sendo que nos Estados do Maranháo e Alagoas ocupam o 90 lugar e no Estado do Acre, o 10? lu$\operatorname{gar}^{23}$.

No panorama nacional, o câncer de colon e reto encontra-se em 50 lugar para o sexo masculino e em 49 lugar para o sexo feminino, considerando-se a distribuição percentual de exames histopatológicos ${ }^{22}$. Constata-se, desta forma, que a distribuição percentual de câncer de colon $\mathrm{e}$ reto apresenta-se com maior freqüência nos grandes centros brasileiros e que aproxima-se dos dados estatísticos encontrados nos países altamente industrializados.

Verifica-se na capital de São Paulo, o maior centro industrial brasileiro, uma área de alto risco da doença comparada a outras capitais, como Recife, que representam áreas de baixo risco.

Os dados apresentados por Walker ${ }^{32}$ (1976) mostram que 10 a $21 \%$ do total de óbitos nos países industrializados são devido ao câncer. Destes, aproximadamente 19 a $20 \%$ são representados pelo câncer de colon.

Estudos realizados, verificando a influên. cia da religião e do fluxo migratório de povos, sugerem haver inter-relaçōes entre a taxa de incidência do câncer do intestino grosso e as condiçōes ambientais das populaçס̄es ${ }^{14,20,21}$, citando os Estados Unidos, como exemplo, que apresentam elevadas taxas de incidência do câncer de colon e reto. Observa-se na região de Utah, que sofre influência religiosa dos Mormons, menores índices de câncer do intestino grosso e que estas taxas asseme. lham-se àquelas observadas nas populaçōes de vegetarianos dos Estados Unidos. Enstrom $^{12}$ (1978) faz referência ao fato de que esta religião recomenda a prática de uma dieta balanceada, tendo como base o uso de grâos integrais, frutas frescas e vegetais e mo. deração no hábito de comer carne.

Burr e Sweetnam ${ }^{8}$ (1978) observaram que as taxas de mortalidade dos vegetarianos americanos e dos adventistas do $70^{\circ} \mathrm{Dia}, \mathrm{cu}$ jos hábitos alimentares apresentam similari. dade, são baixas.

Estudos realizados, por Haenszel e col. ${ }^{14}$, mostram que os japoneses que emigraram para a Califórnia e Hawai passaram a apresentar riscos de câncer de colon, duas a três vezes mais elevado do que aquele registrado no país de origem, fato este provavelmente justificado pela adoção da dieta americana. Aqueles autores ${ }^{14}$ citam dados referentes ao acréscimo de casos de câncer de colon e reto, verificado entre noruegueses, poloneses, portoriquenhos e chineses que imigraram para os Estados Unidos, atingindo o mesmo nível dos americanos brancos.

Os poloneses que imigraram para a Austrália, após a II Guerra Mundial, apresentaram grande aumento nos casos de câncer de colon e reto, atingindo níveis semelhantes ao do povo australiano ${ }^{30}$.

Mass e Modan 22 citam que os judeus ra. dicados em Israel, oriundos do Yemen e norte da África, apresentaram menor risco de câncer intestinal do que os judeus da Europa Ocidental e dos Estados Unidos.

Bremner e Ackerman ${ }^{2}$ (1970) relataram a raridade do câncer do intestino grosso na África e citam a revisão feita no período de 13 anos, em espécimes cirúrgicas de hospital de 2.000 leitos, que revelou somente seis casos de câncer do intestino grosso.

A incidência de câncer do intestino grosso na In dia é mais elevada do que na Âfrica, porém muito mais baixa do que no mundo oci. dental ${ }^{17}$.

Os países industrializados apresentam casos de câncer do intestino grosso, com uma incidência maior que nos países da África, desta forma móstrando diferença no risco existente entre a África como um todo e o ocidente industrializado ${ }^{30,31}$.

Howell $^{17}$, analisando os dados da pesquisa alimentar realizada pela Third National Cancer nos Estados Unidos, mostrou que o Norte, onde as taxas de câncer do colon são mais elevadas (população branca), apresentou maior consumo de carne e gordura do que no sul (população negra), regiáo que apresenta o mais baixo risco de câncer do in. testino em relação às outras regióes. 
LOPES, E. da C. da F. et al. Importância da dieta na epidemiologia do câncer de colon e reto. Rev. Saúde públ., S. Paulo, 18:405-10, 1984.

Estas pesquisas tornam evidente a presen. ça de um fator causal - o fator alimentar caracterizado por uma dieta pobre em fibra e rica em gordura - fortemente ligado à distribuição epidemiológica da doença. $\mathrm{O}$ co. nhecimento do hábito alimentar das popula. ções, associado aos estudos epidemiológicos da doença, apresenta importância relevante na ampliação das pesquisas sobre a carcinogênese do colon e reto, e no Brasil, onde encontramos vários grupos raciais e religiosos $\mathrm{e}$ que apresenta grande fluxo imigratório, estes estudos devem ocupar lugar de destaque.

LOPES, E. da F. et al. [The importance of diet in the epidemiology of cancer of the colon and rectum]. Rev. Saúde públ., S. Paulo, 18:405-10, 1984.

ABSTRACT: Studies have been made to verify the relation between the environmental factor, diet rich in fat and deficient in fibre, and the epidemiologic distribution of cancer of the colon and rectum. The research emphasizes the differences between the diets of the industrialized and the developing countries, and the influence of religion and migratory movements on the diet usualy associated with incidence of the disease.

UNITERMS: Epidemiology. Colonic neoplasms. Rectal neoplasms. Food habits. Diet.

\section{REFERENCLAS BIBLIOGRAFICAS}

1. BARCELOS, L.B. \& PECCIN, D.A. Incidência de mortalidade por câncer no Rio Grande do Sul, Brasil. Rev. Saúde públ., S. Paulo, 17:367-76, 1983.

2. BREMNER, C.G. \& ACKERMAN, L.V. Polyps and carcinoma of the large bowel in the South African Bantu. Cancer, Philadelphia, 26:991-9, 1970.

3. BURKITT, D.P. Epidemiology of cancer of the colon and rectum. Cancer, Philadelphia, 28: 3-13, 1981.

4. BURKITT, D.P. Large-bowel cancer: an epidemiologic jigsaw puzzle. J. Nat. Cancer Inst., 54: 2-6, 1975.

5. BURKITT, D.P. Dietary fiber: is it really helpful? Geriatrics, 37:119-26, 1982.

6. BURKITT, D.P. Western diseases and their emergence related to diet. $S$. Afr. med. J., $61: 1013-5,1982$.

7. BURKITT, D.P.; WALKER, A.R.P. \& PAINTER, N.S. Effect of dietary fibre on stools and transit-times, and its role in the causation of disease. Lancet, 2: 1408-12, 1972.
8. BURR, M.L. \& SWEETNAM, P.M. Vegetaria. nism, dietary fiber, and mortality. Amer. J. clin. Nutr., 36:873-7, 1982.

9. CUMMINGS, J.H. What is fiber? In: Spiller, A. \& Amen, R.J. Fiber in human nutrition. New York, Plenum, 1976. p. 1-30.

10. DIETARY fibre transit-time, faecal bacteria, steroids, and colon cancer in two scandinavian population; Report from the International Agency, for Research on Cancer Intestinal Microecology Group. Lancet, 2 207-11, 1977.

11. DRASAR, B.S. \& IRVING, D. Environmental factors and cancer of the colon and breast. Brit. J. Cancer, 27:167-72, 1973.

12. ENSTROM, JE. Cancer and total mortality among active mormons. Cancer, 42: 194351,1978 .

13. HALLS, J. Bowel content shift during normal defaecation. Proc. roy. Soc. Med., 8: S859. 60,1965 .

14. HAENSZEL, W.; BERG, J.W.; SEGI, M.; KURIHARA, M. \& LOCKE, F.B. Large-bowel cancer in Hawaiian Japanese. J. Nat. Cancer Inst., 51 : 1765-79, 1973. 
LOPES, E. da C. da F. et al. Importância da dieta na epidemiologia do câncer de colon e reto. Rev. Saúde públ., S. Paulo, $18: 405-10,1984$.

15. HARDINGE, M.G.; CHAMBERS, A.C.; CROOKS, H. \& STARE, F. J. Nutritional studies of vegetarians. III. Dietary levels of fiber. Amer. J. clin. Nutr., 6: 523-5, 1958.

16. HELLER, S.N. \& HACKLER, L.R. Changes in the crude fibre content of the American diet. Amer. J. clin. Nutr., 31:1510-4, 1978.

17. HILL, M.J.; DRASAR, B.S.; ARIES, V.; CROWTHER, J.S.; HAWKSWORTH, G. \& WILLIAMS, R.E.O. Bacteria and etiology of cancer large bowel. Lancet, 1:95-100, 1971.

18. HOWELL, M.A. Diet as an etiological factor in the development of cancers of the colon and rectum. J. chron. Dis., 28:67-80, 1975.

19. KRITCHEVSKY, D. Dietary fiber and other dietary factors in hypercholesterolemia. Amer. J. clin. Nutr., 30:979-84, 1977.

20. LYON, J.L. \& SORENSON, A.W. Colon cancer in a lowrisk population. Amer. J. clin. Nutr., 31 : S227-30, 1978.

21. IYON, J.L.; GARDNER, J.W.; KLAUBER, M. R. \& SMART, C.R. Low cancer incidence and mortality in Utah. Cancer, 39:2608-18, 1977.

22. MASS, N. \& MODAN, B. Epidemiological aspects of neoplastic disorders in Israel imigrant population. IV - Cancer of the colon and rectum. J. Nat. Cancer Inst., 42: 529 . $36,1969$.

23. MINISTERIO DA SAODE. Campanha Nacional de Combate ao Câncer. Cancer no Brasil: dados histopatologicos; ed. Rodolfo Brumini. Rio de Janeiro, 1982.

24. PAINTER, N.S. \& BURKITT, D.P. Diverticular disease of the colon: a deficiency disease of Western civilization. Brit. med. J., 2:4504, 1971.

25. REDDY, B.S. Dietary fibre and colon cancer: epidemiologic and experimental evidence. Can. med. Ass. J., Otawa, 123: 850-6, 1980.

26. ROBERTSON, J. Changes in the fibre content of the British diet. Nature, 238: 290-1, 1972.

27. SILVA, M.G.C. da Câncer em Fortaleza: morbi. dade e mortalidade no periodo 1978-80. Fortaleza, Secretaria de Cultura e Desporto, 1982.

28. SOUTHGATE, D.A.T.; BINGHAM, S. \& ROBERTSON, J. Dietary fibre in the British diet. Nature, 274/ 51-2, 1978.
29. SOUZA, J.M.P. de Cancer epidemiology: perspectives in Brazil ard in the city of Săo Pau. lo. In: UICC Conference on Cancer Preven. tion in Developing Countries, $1^{\text {st }}$, Nagoya, 1981. Cancer prevention in developing countries; proceedings. Nagoya, The University of Nagoya Press, 1982.p. 236-44.

30. STASZEWSKI, J. \& HAENSZEL, W. Cancer mortality among the Polish - born in the United States. J. Nat. Cancer Inst., 35 : 2917,1965 .

31. TROWELL, $H$. Definition of dietary fiber and hypotheses that is a protective factor in certain disease. Amer. J. clin. Nutr., 29:417-27, 1976.

32. WALKER, A.R.P. Colon cancer and diet, with special reference to intakes of fat and fiber. Amer. J. clin. Nutr., 29:1417-26, 1976.

33. WALKER, A.R.P.; SEGAL, I. \& HATHORN, S. Dietary fibre and survival. Lancet, 2: $980,1982$.

34. WATERHOUSE, J.; MUIR, C.; CORREA, P. \& POWELL, J., eds. Cancer incidence in five continents. Lyon, International Agency for Research on Cancer, 1976. v. 3 (IARC Scientific Publications, 15).

35. WATERHOUSE, J.; MUIR, C.; SHANMUGARATNAM, K. \& POWELL, J., eds. Cancer incidence in five continents. Lyon, International Agency for Research on Cancer, 1982. v. 4 (IARC Scientific Publications, 42).

36. WYNDER, E.L. \& REDDY, B.S. Studies of large bowel cancer: human leads to experimental applications. J. Nat. Cencer Inst., 50: 1099-106, 1973.

37. WYNDER, E.L. \& REDDY, B.S. Dietary fat and colon cancer. J. Nat. Cancer Inst., 54: 7-10, 1975 .

38. WYNDER, E.L. \& SHIGEMATSU, T. Environmental factors of cancer of the colon and rectum. Cancer, 20:1520-61, 1967. 